\title{
Prologue: 2018 Annual Meeting of the Group for Research and Assessment of Psoriasis and Psoriatic Arthritis (GRAPPA)
}

\author{
Kristina Callis Duffin, Dafna D. Gladman (1), Alice B. Gottlieb, and Niti Goel
}

\begin{abstract}
The 2018 Annual Meeting of the Group for Research and Assessment of Psoriasis and Psoriatic Arthritis (GRAPPA) was held in Toronto, Ontario, Canada, and was attended by rheumatologists, dermatologists, and representatives of biopharmaceutical companies and patients. As in previous years, GRAPPA members held a symposium for trainees to discuss their research in psoriatic disease with experts in the field. Other subjects featured during the annual meeting included GRAPPA-Collaborative Research Network's second annual meeting; the association between psoriatic disease and cardiovascular events; updates from working groups in International Dermatology Outcome Measures (IDEOM) and Outcome Measures in Rheumatology (OMERACT); a 2016 study that benchmarked care in psoriatic arthritis (PsA); the genetic contribution to PsA and strong need for genome-wide association studies on patients with PsA; the GRAPPA Ultrasound Working Group's goal to optimize the evaluation of enthesitis in patients with PsA using ultrasound through the development and validation of new instruments; and an update on GRAPPA's research and educational projects. In this prologue, we introduce the papers that summarize the meeting. (J Rheumatol Suppl. 2019 June;95:1-3; doi:10.3899/jrheum.190112)
\end{abstract}

Key Indexing Terms:

PSORIASIS

PSORIATIC ARTHRITIS

OMERACT

IDEOM

GRAPPA

OUTCOME MEASURES

The 2018 annual meeting of the Group for Research and Assessment of Psoriasis and Psoriatic Arthritis (GRAPPA) was held in July in Toronto, Ontario, Canada, and was attended by investigators in rheumatology and dermatology, representatives of biopharmaceutical companies, and patient

From GRAPPA (Groups for Research and Assessment of Psoriasis and Psoriatic Arthritis), Seattle, Washington; University of Utah, Salt Lake City, Utah; Department of Dermatology, Icahn School of Medicine at Mount Sinai, New York, New York; Kezar Life Sciences, San Francisco, California; Duke University School of Medicine, Durham, North Carolina, USA; University of Toronto; Krembil Research Institute, Toronto Western Hospital, Toronto, Ontario, Canada.

As part of the supplement series GRAPPA 2018, this report was reviewed internally and approved by the Guest Editors for integrity, accuracy, and consistency with scientific and ethical standards.

Financial support to enable these meetings was provided in 2018 by AbbVie, Amgen, Bristol-Myers Squibb, Celgene, Janssen, Eli Lilly \& Company, Novartis, Pfizer, and UCB. In addition, our Innovation Partners in 2018 were LEO Pharma, Sienna Biopharmaceuticals, and Sun Pharma.

K. Callis Duffin, MD, University of Utah, Salt Lake City, and President, GRAPPA; D.D. Gladman, MD, FRCPC, Professor of Medicine, University of Toronto, and Senior Scientist, Krembil Research Institute, Toronto Western Hospital, and Co-chair, GRAPPA Publication Committee; A.B. Gottlieb, MD, PhD, Clinical Professor of Dermatology, Department of Dermatology, Icahn School of Medicine at Mount Sinai, and Co-chair, GRAPPA Publication Committee; N. Goel, MD, Patient Research Partner, Chief Medical Officer, Kezar Life Sciences, and Adjunct Assistant

Professor, Duke University School of Medicine, and Co-chair, GRAPPA Publication Committee.

Address correspondence to Dr. K. Callis Duffin, University of Utah, 4 A330 Dermatology SOM, 30 North 1900 East, Salt Lake City, Utah 84132, USA.

E-mail: kristina.callis@hsc.utah.edu research partners (PRP; Table 1 and Table 2) who discussed individual and collaborative research and educational initiatives in psoriasis and psoriatic arthritis (PsA). Currently, there are 951 worldwide GRAPPA members, including investigators in rheumatology and dermatology $(n=650)$, representatives of biopharmaceutical companies $(n=207)$, PRP $(n=12)$, and other members $(n=82)$. Reports of previous yearly meetings have been published elsewhere $\mathrm{e}^{1,2,3,4,5,6,7,8,9}$.

A Trainees Symposium was held prior to the annual meeting in which rheumatology and dermatology researchertrainees from Europe, North America, and South America who are current GRAPPA members or who were nominated by GRAPPA members presented and discussed their studies with experts in the field. A total of 54 abstracts were submitted and ranked by a committee of reviewers. Five trainees with the highest-scored abstracts were invited to deliver oral presentations; all trainees presented posters that outlined key aspects of their research. Dr. Christopher T. Ritchlin (rheumatologist, Rochester, New York, USA) and Dr. Wolf-Henning Boehncke (dermatologist, Geneva, Switzerland) co-chaired the symposium in which GRAPPA members discussed the findings presented by trainees and suggested how trainees might further their current research projects $^{10}$.

The GRAPPA-Collaborative Research Network (CRN) held its second annual meeting following the GRAPPA 2018 annual meeting. The GRAPPA-CRN intends to launch and

Personal non-commercial use only. The Journal of Rheumatology Copyright $\subset$ 2019. All rights reserved. 
Table 1. GRAPPA 2018 annual meeting attendance.

\begin{tabular}{lc}
\hline Participant Type & Total, $\mathrm{n}$ \\
\hline Dermatologist & 24 \\
Rheumatologist & 112 \\
PRP & 10 \\
Trainees & 23 \\
Industry & 52 \\
Total & 221 \\
\hline
\end{tabular}

GRAPPA: Group for Research and Assessment of Psoriasis and Psoriatic Arthritis; PRP: Patient research partner.

Table 2. GRAPPA executive and steering committee membership.

\begin{tabular}{lc}
\hline Executive Committee & Position \\
\hline Kristina Callis Duffin & President \\
Oliver FitzGerald & Vice president and president-elect \\
Philip S. Helliwell & Immediate past president \\
Arthur Kavanaugh & Co-treasurer \\
Philip Mease & Co-treasurer \\
Alice B. Gottlieb & Member at-large \\
\hline Publications Committee & Position \\
\hline Dafna D. Gladman & Co-chair \\
Alice B. Gottlieb & Co-chair \\
Niti Goel & Co-chair
\end{tabular}

\begin{tabular}{|c|c|}
\hline Steering Committee & City, Country \\
\hline Ade Adebajo & Sheffield, UK \\
\hline April W. Armstrong & Los Angeles, CA, USA \\
\hline Kristina Callis Duffin & Salt Lake City, UT, USA \\
\hline Alberto Cauli & Cagliari, Italy \\
\hline Vinod Chandran & Toronto, ON, Canada \\
\hline Laura C. Coates & Oxford, UK \\
\hline Atul A. Deodhar & Portland, OR, USA \\
\hline Oliver FitzGerald & Dublin, Ireland \\
\hline Amit Garg & Manhasset, NY, USA \\
\hline Dafna D. Gladman & Toronto, ON, Canada \\
\hline Niti Goel & Durham, NC, USA \\
\hline Alice B. Gottlieb & New York, NY, USA \\
\hline Philip S. Helliwell & Leeds, UK \\
\hline Elaine Husni & Cleveland, OH, USA \\
\hline Arthur Kavanaugh & La Jolla, CA, USA \\
\hline Philip Mease & Seattle, WA, USA \\
\hline Joseph F. Merola & Boston, MA, USA \\
\hline Peter Nash & Brisbane, Queensland, Australia \\
\hline Alexis Ogdie & Philadelphia, PA, USA \\
\hline Ana-Maria Orbai & Baltimore, MD, USA \\
\hline Denis O’Sullivan & Dublin, Ireland \\
\hline Christopher T. Ritchlin & Rochester, NY, USA \\
\hline Jose Scher & New York, NY, USA \\
\hline Stefan Siebert & Glasgow, Scotland, UK \\
\hline Evan Siegel & Rockville, MD, USA \\
\hline Enrique Soriano & Buenos Aires, Argentina \\
\hline Vibeke Strand & Portola Valley, CA, USA \\
\hline Diamant Thaçi & Lubeck, Germany \\
\hline
\end{tabular}

GRAPPA: Group for Research and Assessment of Psoriasis and Psoriatic Arthritis. secure funding for 3 pilot projects related to psoriatic disease, i.e., PsA and cutaneous psoriasis (PsC). The first project will aim to identify biomarkers that predict treatment response in PsA and PsC. The second, the PsA Biomarkers for Joint Damage (BioDAM) project, will seek to determine the independent predictive ability of serum biomarkers for joint damage in PsA. The third project will aim to identify predictors of the development of PsA among patients with PsC. These pilot projects will prompt the development of clinical protocols to operate across participating centers, lead to the development of standard operating procedures for the collection and transport of biosamples across international borders, and begin to establish administrative and managerial structures for the $\mathrm{CRN}^{11}$.

In a symposium on the association between psoriatic disease and cardiovascular (CV) events, several GRAPPA members discussed recent data about the association between psoriasis and vascular inflammation, the use of coronary angiogram to investigate $\mathrm{CV}$ outcomes, new approaches for $\mathrm{CV}$ risk stratification, and the shared pathomechanisms of psoriasis and atherosclerosis ${ }^{12}$.

The International Dermatology Outcome Measures (IDEOM) Psoriasis Working Group presented an overview of its efforts to enhance clinical care and research in both the clinical setting as well as in clinical trials for psoriasis. The group discussed the results of a Delphi survey conducted in collaboration with the American Academy of Dermatology to agree on a unique physician-reported global assessment to measure the quality of care delivered to patients with psoriasis and other chronic inflammatory dermatoses. The group then summarized its efforts to select outcome measures for "PsA Symptoms" and "Treatment Satisfaction," 2 of the domains of the psoriasis core domain set that were established by IDEOM. Last, the Psoriasis and Psoriatic Arthritis Clinics Multicenter Advancement Network (PPACMAN) presented an update on its clinical, educational, and research missions to foster the development of combined clinics for psoriatic disease to increase disease awareness and accelerate management ${ }^{13}$.

The GRAPPA-Outcome Measures in Rheumatology (OMERACT) PsA Working Group reported at the 2018 GRAPPA annual meeting on the outcome of the OMERACT 2018 Conference in Terrigal, Australia. The working group presented the endorsement of the $66 / 68$ joint count for the assessment of peripheral arthritis and the provisional endorsement of the PsA Impact of Disease 12 questionnaire for the assessment of PsA-specific health-related quality of life in PsA randomized controlled trials and observational studies. The group plans to seek OMERACT endorsement for outcome measures that address the domains of physical function and structural damage following the OMERACT Filter 2.1 methodology ${ }^{14}$.

In a symposium discussing a 2016 study that benchmarked care in PsA that was conducted by GRAPPA and KPMG LLP, 
a key finding was that centers do not usually have processes in place to measure the effect of improved quality of care. During the symposium, several GRAPPA members presented an initial set of concise indicators for best practice in PsA resulting from an integrative literature review, electronic survey, and consensus discussions among an international panel of dermatology and rheumatology experts. These preliminary indicators can serve as benchmarks for delivering quality care in the clinical setting and may enhance the care of patients with psoriatic disease worldwide ${ }^{15}$.

In a symposium on the genetic contribution to PsA, several GRAPPA members discussed the strong need for genome-wide association studies on patients with PsA, including PsA-weighted or specific variants, and a need for a better understanding of the relevance of HLA alleles in disease expression ${ }^{16}$.

In a symposium on the GRAPPA Ultrasound Working Group's recent work, the group discussed how ultrasound (US) could improve the accuracy of clinical enthesitis assessment. The group set a goal to optimize the evaluation of enthesitis in patients with PsA using US through the development and validation of new instruments that use a combined data-driven and expert opinion-driven approach ${ }^{17}$.

Members received updates on several ongoing educational and research efforts. Among them were updates on GRAPPA's PRP; educational initiatives; research efforts, including the trainee symposium, pilot research grants, and the CRN; treatment recommendations; and additional efforts related to advancing the understanding of disease aspects, including the axial project, early PsA systematic literature review, and imaging ${ }^{18}$.

At the conclusion of the GRAPPA meeting, members discussed future action items in a business meeting. The next annual meeting will be held in Paris, France, in July 2019.

\section{ACKNOWLEDGMENT}

Special thanks goes to Pam Love for her tireless organizational efforts, which kept the meeting running smoothly.

\section{REFERENCES}

1. Mease PJ, Gladman DD. Prologue: 2009 Group for Research and Assessment of Psoriasis and Psoriatic Arthritis (GRAPPA). J Rheumatol 2011;38:522-5.
2. Mease PJ, Gladman DD. Prologue: 2010 Group for Research and Assessment of Psoriasis and Psoriatic Arthritis (GRAPPA). J Rheumatol 2012;39:391-3.

3. Mease PJ, Gladman DD. Prologue: 2011 Group for Research and Assessment of Psoriasis and Psoriatic Arthritis (GRAPPA). J Rheumatol 2012;39:2181-3.

4. Mease PJ, Boehncke WH, Gladman DD. Prologue: 2012 annual meeting of the Group for Research and Assessment of Psoriasis and Psoriatic Arthritis (GRAPPA). J Rheumatol 2013;40:1407-9.

5. Boehncke WH, Gladman DD. Prologue: 2013 annual meeting of the Group for Research and Assessment of Psoriasis and Psoriatic Arthritis (GRAPPA). J Rheumatol 2014;41:1194-6.

6. Boehncke WH, Gladman DD. Prologue: 2014 annual meeting of the Group for Research and Assessment of Psoriasis and Psoriatic Arthritis (GRAPPA). J Rheumatol 2015;42:2011-13.

7. Boehncke WH, Gladman DD, Helliwell PS. Prologue: 2015 annual meeting of the Group for Research and Assessment of Psoriasis and Psoriatic Arthritis (GRAPPA). J Rheumatol 2016;43:949-51.

8. Helliwell PS, Gladman DD, Gottlieb AB. Prologue: 2016 annual meeting of the Group for Research and Assessment of Psoriasis and Psoriatic Arthritis (GRAPPA). J Rheumatol 2017;44:658-60.

9. Helliwell PS, Gladman DD, Gottlieb AB. Prologue: 2017 annual meeting of the Group for Research and Assessment of Psoriasis and Psoriatic Arthritis (GRAPPA). J Rheumatol Suppl. 2018 June; 94:1-3.

10. Ford A, Mascia E, Boehncke W, Ritchlin C. GRAPPA Trainees Symposium 2018: a report from the GRAPPA 2018 annual meeting. J Rheumatol Suppl. 2019 June;95:4-10.

11. Jadon D, Chandran V, Ogdie A, Pennington S, Stober C, Gladman DD, et al. Proceedings of the 2018 GRAPPA Collaborative Research Network (CRN) meeting. J Rheumatol Suppl. 2019 June;95:11-19.

12. Eder L, Dey A, Joshi A, Boehncke W, Mehta N, Szentpetery A. Cardiovascular diseases in psoriasis and psoriatic arthritis. J Rheumatol Suppl. 2019 June;95:20-7.

13. Perez-Chada L, Merola J, Armstrong A, Gottlieb A. Report of the skin research workgroups from the GRAPPA 2018 annual meeting. J Rheumatol Suppl. 2019 June;95:28-32.

14. Leung YY, Orbai A, Ogdie A, Coates L, de Wit M, Callis Duffin K, et al. The GRAPPA-OMERACT Psoriatic Arthritis Working Group at the 2018 annual meeting: report and plan for completing the core outcome measurement set. J Rheumatol Suppl. 2019 June;95:33-7.

15. Helliwell P, Favier G, Gladman D, Soriano E, Kirkham B, Coates L, et al. Best practice indicators in psoriatic disease care. J Rheumatol Suppl. 2019 June;95:38-45.

16. O'Rielly D, Jani M, Rahman P, Elder J. The genetics of psoriasis and psoriatic arthritis. J Rheumatol Suppl. 2019 June;95:46-50.

17. Eder L, Kaeley G, Aydin S. The GRAPPA sonographic enthesitis workshop. J Rheumatol Suppl. 2019 June;95:51-3.

18. Goel N, FitzGerald O, Gladman D, Helliwell P, Kavanaugh A, Maksymowych W, et al. GRAPPA 2018 project report. J Rheumatol Suppl. 2019 June;95:54-7. 